\title{
Periodic Quantum Tunneling and Parametric Resonance with Cigar-Shaped Bose-Einstein Condensates
}

\author{
L. Salasnich ${ }^{1}$, A. Parola ${ }^{2}$ and L. Reatto ${ }^{1}$ \\ ${ }^{1}$ Istituto Nazionale per la Fisica della Materia, Unità di Milano, \\ Dipartimento di Fisica, Università di Milano, \\ Via Celoria 16, 20133 Milano, Italy \\ ${ }^{2}$ Istituto Nazionale per la Fisica della Materia, Unità di Como, \\ Dipartimento di Scienze Fisiche, Università dell'Insubria, \\ Via Valeggio 11, 23100 Como, Italy
}

\begin{abstract}
We study the tunneling properties of a cigar-shaped Bose-Einstein condensate by using an effective 1D nonpolynomial nonlinear Schrödinger equation (NPSE). First we investigate a mechanism to generate periodic pulses of coherent matter by means of a Bose condensate confined in a potential well with an oscillating height of the energy barrier. We show that is possible to control the periodic emission of matter waves and the tunneling fraction of the Bose condensate. We find that the number of emitted particles strongly increases if the period of oscillation of the height of the energy barrier is in parametric resonance with the period of oscillation of the center of mass of the condensate inside the potential well. Then we use NPSE to analyze the periodic tunneling of a Bose-Einstein condensate in a double-well potential which has an oscillating energy barrier. We show that the dynamics of the Bose condensate critically depends on the frequency of the oscillating energy barrier. The macroscopic quantum self-trapping (MQST) of the condensate can be suppressed under the condition of parametric resonance between the
\end{abstract}


frequency of the energy barrier and the frequency of oscillation through the barrier of the very small fraction of particles which remain untrapped during MQST. 


\section{INTRODUCTION}

In the last few years macroscopic quantum tunneling (MQT) with dilute Bose-Einstein condensates of alkali-metal atoms has been the subject of many theoretical [1-4] and experimental [5-7] studies. In a recent paper we have suggested a mechanism to generate pulses of coherent matter by using MQT of a falling and bouncing condensate [8]. In the first part of this paper we propose another, simpler, mechanism to produce periodic atomic wave-packets by means of MQT of a Bose condensate through the barrier of a potential well. In our theoretical investigation we consider cigar-shaped Bose condensates so that we can use an effective 1D nonpolynomial nonlinear Schrödinger equation, which we have shown to be quite accurate when the aspect ratio is larger than $3[9,10]$. We consider a Bose-Einstein condensate confined in the vertical axial direction by two Gaussian optical barriers and in the transverse direction by a magnetic or optical harmonic potential. By periodically changing the height of the lower-lying Gaussian barrier it is possible to generate periodic waves of coherent matter: only when the height of the energy barrier is sufficiently small a consistent fraction of condensed atoms can tunnel. We show that it is possible to control the period of emission of the matter waves and the tunneling probability. We find that the emission probability can be strongly enhanced by using the parametric resonance between the oscillation of the height of the energy barrier and the oscillation of the center of mass of the condensate inside the potential well (resonance-induced coherent emission).

In the second part of the paper we study another problem of tunnelling, the one of trapped particles in a double potential well. In particular, we investigate the effect of a periodically varying barrier in the tunneling of the Bose condensate. The periodic oscillations of a Bose-Einstein condensate in a double-well has been the subject of many papers [1-4] but, to our knowledge, the role of a time-dependent energy barrier has not been analyzed. We investigate the problem using our effective 1D nonpolynomial Schrödinger equation and also the two-mode classical-like equations introduced by Smerzi et al [1]. The numerical results obtained with the two methods are in quantitative agreement. In particular, we find that 
the phenomenon of macroscopic quantum self-trapping of the condensate can be controlled (reduced or suppressed) by the frequency of oscillation of the double-well energy barrier again via parametric resonance.

\section{MAPPING FROM 3D TO 1D}

It is well know that the 3D Gross-Pitaevskii equation (GPE) is accurate in describing the Bose-Einstein condensate of a dilute boson gas at temperatures well below the transition temperature, where thermal excitations can be neglected [11]. Often the condensates produced in experiments are cigar-shaped and this suggests to map the 3D GPE into an effective 1D equation, which simplifies greatly the solution of the equation. This problem is not trivial due to the nonlinearity of the GPE. By using a variational approach, we have recently derived an effective time-dependent 1D nonpolynomial nonlinear Schrödinger equation (NPSE) which describes very accurately cigar-shaped Bose condensates $[9,10]$. We assume that the condensate is confined by a magnetic (or optical) harmonic potential with frequency $\omega_{\perp}$ and harmonic length $a_{\perp}=\left(\hbar / m \omega_{\perp}\right)^{1 / 2}$ in the transverse direction and by a generic potential $V(z)$ in the axial one. The total wave function of the condensate is $\psi(x, y, z, t)=f(z, t) g(x, y, t)$, where the transverse wave-function $g(x, y, t)$ is a Gaussian

with the width $\eta$ such that $\eta^{2}=a_{\perp}^{2} \sqrt{1+2 a_{s} N|f|^{2}}$ with $a_{s}$ the s-wave scattering length [10]. The NPSE is given by

$$
\begin{aligned}
& i \hbar \frac{\partial}{\partial t} f=\left[-\frac{\hbar^{2}}{2 m} \frac{\partial^{2}}{\partial z^{2}}+V(z)+\frac{g N}{2 \pi a_{\perp}^{2}} \frac{|f|^{2}}{\sqrt{1+2 a_{s} N|f|^{2}}}\right. \\
& \left.+\frac{\hbar \omega_{\perp}}{2}\left(\frac{1}{\sqrt{1+2 a_{s} N|f|^{2}}}+\sqrt{1+2 a_{s} N|f|^{2}}\right)\right] f
\end{aligned}
$$

where $f(z, t)$ is the axial macroscopic wave function of the condensate, $g=4 \pi \hbar^{2} a_{s} / m$ is the scattering amplitude and $N$ is the number of condensed bosons and the function $f(z, t)$ is normalized to one.

We observe that in the weakly-interacting limit $a_{s} N|f|^{2}<<1$, NPSE reduces to a 1D 
GPE with the nonlinear coefficient $g^{\prime}$ given by $g^{\prime}=g /\left(2 \pi a_{\perp}^{2}\right)$. Instead, in the stronglyinteracting limit, NPSE becomes a nonlinear Schödinger equation with the nonlinear term proportional to $|f| f$.

In paper [10] we have tested the accuracy of the NPSE in the determination of the groundstate and collective oscillations of the condensate with axial harmonic confinement and also in the description of tunneling through a Gaussian barrier. In particular, we have compared NPSE with the the full 3D GPE. The conclusion is that NPSE is very accurate when the aspect ratio in larger than 3 and gives better results than all other effective approaches recently proposed $[12,13]$.

\section{PERIODIC EMISSION OF MATTER WAVES}

In a previous paper [8] we have analyzed the pulsed quantum tunneling of a Bose condensate falling under gravity and scattering on a Gaussian barrier that model a mirror of far-detuned sheet of light. In this section we study another mechanism which produces periodic emission of matter waves by means of the quantum tunneling of a Bose condensate. We consider a cigar-shaped condensate confined by a harmonic potential in the transverse direction and under the action of the following external potential in the vertical axial direction:

$$
V(z)=V_{1} e^{-\left(z-z_{1}\right)^{2} / \sigma^{2}}+V_{2} e^{-\left(z-z_{2}\right)^{2} / \sigma^{2}}+m g z .
$$

Thus, in addition to the gravity potential $m g z$, there are two Gaussian functions that model a confining potential well. The shape of this potential is shown in Figure 1. Such a configuration can be experimentally obtained by using two blue-detuned laser beams (perpendicular to the axial direction) which are modelled by the two Gaussian potentials. By varying the intensity of the lower-lying laser beam one controls the height of the energy barrier and therefore the tunneling probability. In particular we consider the case in which the height $V_{2}$ of the lower barrier is a sinusoidal function of time. As an example we consider a BoseEinstein condensate of ${ }^{23} \mathrm{Na}$ atoms with a scattering length $a_{s}=30 \AA$. For the frequency $\omega_{\perp}$ 
of the axial harmonic confinement we choose $\omega_{\perp}=2 \pi \mathrm{kHz}$. Setting $\omega_{z}=\omega_{\perp} / 10$, we measure lengths in units $a_{z}=\left(\hbar / m \omega_{z}\right)^{1 / 2}$, time in units $\omega_{z}^{-1}$ and energy in units $\hbar \omega_{z}$. In these units the period of small oscillations around the minimum of the potential well (2) close to one. The NPSE is integrated by using a finite-difference predictor-corrector method. The initial wave function of the condensate is found by solving the equation with imaginary time.

First we calculate the "ground-state" of the system by choosing $V_{1}=1000, V_{2}=900$, $z_{1}=17, z_{2}=24$ and $\sigma=1.6$. Then we reduce the energy barrier $V_{1}$ studying the time evolution and tunneling of the condensate through the potential well. The results are shown in Figure 2. There is a periodic tunneling of matter waves and the period of tunneling is proportional to the period of small oscillations of the center of mass of the condensate around the minimum of the axial external potential $V(z)$. To compare the chemical potential of the Bose condensate with the energy barrier $V_{1}$, the effective chemical potential $\mu_{\text {eff }}$ of the Bose condensate is obtained from the full one by subtracting not only the transverse energy per particle but also the gravitational energy per particle.

A simpler mechanism to generate periodic pulses is to have the lower-lying Gaussian barrier with a periodic height given by:

$$
V_{1}(t)=V_{a}+V_{b} \sin \left(\frac{2 \pi}{\tau} t\right),
$$

where $\tau$ is the period of oscillation. We choose $V_{a}=300$ and $V_{b}=200$. In Figure 3 we plot the axial density profile $\rho(z)$ of the matter-waves coming out from the potential well with $\tau=1.5$. Figure 3 shows that, as expected, the emission of pulses is periodic.

We find that both the period of emission and the tunneling fraction $P_{T}$ depend on $\tau$. For very small values of $\tau$, the fraction of ejected particles is quite small because the height of the energy barrier oscillates very quickly. On the other hand, for large values of $\tau$ the fraction $P_{T}$ is again very small. In the inset of Figure 4 the tunneling probability $P_{T}$ is shown as a function of $\tau$ for a Bose condensate with $N=10^{3}$ and $N=10^{4}$ atoms. The emission probability has its absolute maximum near $\tau=0.8$, that is the average period of the oscillations of the center of mass of the condensate inside the potential well. 
The emission probability $P_{T}$ grows by increasing the number $N$ of atoms and it is strongly enhanced by setting the oscillating barrier at the resonance condition. Nevertheless, by plotting the ratio $P_{T} / P_{T}^{0}$ as a function of $\tau$, where $P_{T}^{0}$ is the tunneling probability with $V_{1}(t)=V_{a}-V_{b}=100$, one finds that the resonance is suppressed by the interaction, i.e. by increasing $N$ (see Figure 4). Note that in the non-interacting case $\left(N=0\right.$ and $\left.\mu_{\text {eff }}=21.88\right)$ one finds $P_{T}^{0}=1.11 \times 10^{-4}$, but $P_{T}=0.35$, thus the tunneling ratio $P_{T} / P_{T}^{0}$ is larger than $3 \times 10^{3}$.

The enhancement of the emission probability of coherent matter near $\tau=0.8$ has a classical explanation. It corresponds to the condition of resonance between the oscillation of the energy barrier and the oscillation of the center of mass of the condensate. Such an effect is clearly shown in Figure 5, where we plot the axial coordinate and the phase-space portrait of a classical particle under the action of the potential (2). The dynamics of the classical particle is obtained by solving the Hamilton equations with a 4th-order RungeKutta method. In Figure 5, for $\tau=0.75$ the system is close to the resonance condition: the periodic energy of the barrier is pumped into the energy of the classical particle and the particle is able to escape from the trap. Far from the resonance condition, the periodic energy of the barrier is not fully transferred to the energy of the classical particle, so the particle remains confined. Close to the resonance condition the phenomenon of coherent emission of atoms is no more due to quantum tunneling but to the parametric resonance [14] between the period of oscillation of the center of mass of the condensate and the period of oscillation of the energy barrier.

Because of the presence of an oscillating barrier of period $\tau$, the period $\tilde{\tau}_{o s c}$ of oscillation of the center of mass of the condensate follows the law $\tilde{\tau}_{\text {osc }}=\tau_{\text {osc }}(1+\epsilon \sin (2 \pi t / \tau))$, where $\tau_{\text {osc }}$ is the period of oscillation of the center of mass in the absence of an oscillating barrier and $\epsilon$ represents the amplitude of the periodic perturbation. Text-books [14] predict parametric resonance for $\tau=(n / 2) \tau_{\text {osc }}$ with $n$ an integer number. In fact, as shown in Figure 4, there are other local maxima in the plot of the emission fraction whose positions follow this resonant condition formula. 


\section{PERIODIC TUNNELING IN A OSCILLATING DOUBLE-WELL POTENTIAL}

In this section we investigate the dynamics of a Bose-Einstein condensate of ${ }^{23} \mathrm{Na}$ atoms in a double-well trap given by a harmonic anisotropic potential plus a Gaussian barrier along the $z$ axis, which could model the effect of a laser beam perpendicular to the long axis of the condensate. In our model the height of the Gaussian barrier is periodic and performs small oscillations around its mean value.

The Bose condensate is confined by a harmonic potential in the transverse direction while the external potential in the horizontal axial direction is given by:

$$
V(z, t)=V_{1}(t) e^{-z^{2} / \sigma^{2}}+\frac{1}{2} m \omega_{z}^{2} z^{2}
$$

where again

$$
V_{1}(t)=V_{a}+V_{b} \sin \left(\frac{2 \pi}{\tau} t\right)
$$

We perform the numerical integration of NPSE with the potential (4) using the $z$-harmonic oscillator units with $\omega_{z}=\omega_{\perp} / 10$ and $\omega_{\perp}=2 \pi \mathrm{kHz}$. The shape of this double-well external potential is shown in Figure 6. Such a configuration can be experimentally obtained by using a procedure similar to that described in the previous section (see also [15]).

First we calculate the "ground-state" of the system fully confined in the right well by choosing $V_{1}=20$ and $V_{2}=0$. Then we reduce the energy barrier $V_{1}$ studying the time evolution and periodic tunneling of the condensate through the Gaussian barrier. In Figure 7 we plot the fraction $P_{L}$ of ${ }^{23} \mathrm{Na}$ atoms in the left well as a function of time $t$, choosing $V_{1}=6.5$ and $V_{2}=0$. The behavior of $P_{L}(t)$ depends on the inter-atomic energy of the condensate. As shown in Figure 7, for small values of the inter-atomic strength $N a_{s} / a_{z}$ the condensed cloud oscillates between the two wells, while for larger values of $N a_{s} / a_{z}$ the condensate remains self-trapped. Thus, for large values of the inter-atomic interaction, only a small part of atoms oscillates between the two wells while the main part of the atomic condensate remains confined in the right well. This phenomenon, called macroscopic quantum self-trapping (MQST), has been recently predicted by Smerzi et al. [1]. 
Smerzi et al. [1] have found that the time-dependent behavior of the condensate in the tunneling energy range can be described by the two-mode equations

$$
\dot{\zeta}=-\sqrt{1-\zeta^{2}} \sin \phi, \quad \dot{\phi}=\Lambda \zeta+\frac{\zeta}{\sqrt{1-\zeta^{2}}} \cos \phi
$$

where $\zeta=\left(N_{1}-N_{2}\right) / N$ is the fractional population imbalance of the condensate in the two wells, $\phi=\phi_{1}-\phi_{2}$ is the relative phase (which can be initially zero), and $\Lambda=2 E_{I} / E_{T}$ with $E_{I}$ the inter-atomic energy and $E_{T}$ the tunneling energy, i.e. the kinetic+potential energy splitting between the ground state and the quasi-degenerate odd first excited state of the GP equation. Note that $E_{T}=\hbar \omega_{0}$, where $\omega_{0}$ is the oscillation frequency of the Bose condensate between the two wells when the inter-atomic interaction is zero $\left(E_{I}=0\right)$. For a fixed $\Lambda(\Lambda>2)$, there exists a critical $\zeta_{c}=2 \sqrt{\Lambda-1} / \Lambda$ such that for $0<\zeta<<\zeta_{c}$ there are Josephson-like oscillations of the condensate with period $\tau_{J}=\tau_{0} / \sqrt{1+\Lambda}$, where $\tau_{0}=2 \pi / \omega_{0}$. But for $\zeta_{c}<\zeta \leq 1$ there is macroscopic quantum self-trapping (MQST) of the condensate: even if the populations of the two wells are initially set in an asymmetric state $(\zeta(0) \neq 0)$ they maintain, on the average, the original population imbalance with a very small periodic transfer of particles through the barrier with period $\tau_{S T}=\tau_{0} / \sqrt{2\left(\Lambda H_{0}-1\right)}$, where $H_{0}=\Lambda \zeta(0)^{2} / 2-\sqrt{1-\zeta(0)^{2}} \cos \phi(0)[1]$. Our NPSE numerical results of Figure 7 confirm the two-mode approximation (Eq. (6)) prediction: the fully imbalanced $(\zeta(0)=1)$ Bose-Einstein condensate approaches a self-trapped regime by increasing $N a_{a} / a_{z}$, i.e. the inter-atomic energy.

It is interesting to see what happens if we include a periodic oscillation of the barrier $\left(V_{2} \neq 0\right)$. As shown in Figure 8, where we plot the condensate fraction $P_{L}(t)$ in the lefthanded well obtained by numerically integrating the NPSE, the presence of an oscillating barrier of period $\tau$ can break or simply reduce the MQST of period $\tau_{S T}$. In particular, in Figure 8 it is shown that if $\tau=\tau_{S T} / 2$ (parametric resonance condition [14]) then the MQST is broken; instead, if $\tau=2 \tau_{S T}$ the MQST is not broken but there is a larger periodic transfer of particles through the barrier.

This phenomenon can be investigated by using the two-mode approximation of Eq. (4). 
In this case, the presence of a oscillating barrier can be modelled by a time-dependent $\Lambda$ : e.g. $\Lambda(t)=\Lambda_{0}(1+\epsilon \sin (2 \pi t / \tau))$. In Figure 9 we plot the population imbalance $\zeta(t)$ and its phase-space portrait $(\zeta(t), \dot{\zeta}(t))$ with $\Lambda_{0}=25, \zeta(0)=0.6, \phi(0)=0$. Also in this case the MQST is strongly affected by the parametric resonance between the period $\tau$ of MQST oscillation and the period $T$ of oscillation of $\Lambda$ (i.e. the energy barrier). As shown in Figure 10, at the parametric resonance condition $\tau=\tau_{S T} / 2$ and with a sufficiently large perturbation $(\epsilon=0.2)$ the system escapes from the self-trapping configuration. We have verified that for this system the general parametric resonance condition $\tau=(n / 2) \tau_{S T}[14]$ works quite well at small $n$ (even and odd).

Recently the parametric excitation of cold trapped atoms in far-off-resonance optical lattices has been experimentally obtained by modulating the potential depth $[16,17]$. In these experiments the parametric resonance results in heating or losses for the trapped atoms and it can be studied to determine the spring constant of the periodic optical potential [17]. Another kind of parametric resonance, namely the parametric resonance between the collective oscillations of a trapped Bose-Einstein condensate and the oscillations of the confining harmonic trapping potential has been demonstrated with numerical simulations by Kevrekidis, Bishop and Rasmussen [18]. They have shown that a weak harmonic modulation of the confining potential can cause an anomalously large amplitude in the collective oscillations of the Bose condensate. Actually, a similar effect can be obtained without parametric resonance by imposing particular values of anisotropy to the trapping potential: for such values different collective modes of the condensate are in resonance [19].

\section{CONCLUSIONS}

We have studied the macroscopic quantum tunneling of a cigar-shaped Bose-Einstein condensate confined by a harmonic potential in the transverse direction and tunneling through an energy barrier in the axial direction. The time-evolution of the macroscopic wave function

of the condensate is obtained by using an effective 1D nonpolynomial nonlinear Schrödinger 
equation.

First we have investigated a Bose condensate confined in the vertical axial direction by two Gaussian barriers that model a potential well. By periodically changing the height of the lower-lying Gaussian barrier it is possible to generate periodic waves of coherent matter: only when the height of the energy barrier is sufficiently small a consistent fraction of condensed atoms can tunnel. We show that it is possible to control the period of emission of the matter waves and the tunneling probability. We have also found a resonance-induced coherent emission of atoms: the emission probability is strongly enhanced if the period of oscillation of the height of the energy barrier is in parametric resonance with period of oscillation of the center of mass of the condensate inside the potential well.

Then we have studied the periodic tunneling and the quantum self-trapping of a BoseEinstein condensate in a double-well potential with an oscillating energy barrier. We have used our effective 1D nonpolynomial Schrödinger equation and also the two-mode classicallike equations. We have found that the macroscopic quantum self-trapping of a Bose condensate can be reduced and also suppressed by changing the frequency of the oscillating barrier. In particular, the system escapes from the self-trapping configuration if the the period of oscillation of the double-well energy barrier and the period of MQST oscillations of the condensate satisfies the parametric resonance condition.

The parametric driving of Bose-Einstein condensates can be obtained by current experiments using optical dipole forces with far-detuned laser beams [20]. By varying the intensity of the laser beams one can control the height of the energy barrier that confines the condensed sample. The experimental investigation of the physical configurations we have considered in this paper may contribute to the realization of novel phenomena: controlled periodic emission in atom lasers and breaking of macroscopic quantum self-trapping induced by parametric resonance. 


\section{REFERENCES}

[1] Smerzi A, Fantoni S, Giovannazzi S, and Shenoy S R 1997 Phys. Rev. Lett. 79 4950; Raghavan S, Smerzi A, Fantoni S, and Shenoy S R 1999 Phys. Rev. A 59620.

[2] Milburn G J, Corney J, Wright E, and Walls D F 1997 Phys. Rev. A 554318

[3] Zapata I, Sols F, and Leggett A J 1998 Phys. Rev. A 57 R28

[4] Salasnich L, Parola A, and Reatto L 1999 Phys. Rev. A 60 4171; Pozzi B, Salasnich L, Parola A, and Reatto L 2000 Eur. Phys. J. D 11367

[5] B.P. Anderson and M. Kasevich 1999 Science 2821686

[6] Cataliotti F S, Burger S, Fort C, Maddaloni P, Trombettoni A, Smerzi A, and Inguscio M 2001 Science 293843

[7] Morsch O, Muller J H, Cristiani M, Ciampini D, and Arimondo E 2001 Phys. Rev. Lett. 87140402

[8] Salasnich L, Parola A, and Reatto L 2001 Phys. Rev. A 64023601

[9] Salasnich L 2002 Laser Physics 12198

[10] Salasnich L, Parola A, and Reatto L 2002 Phys. Rev. A 65043614

[11] Gross E P 1961 Nuovo Cimento 20 454; Pitaevskii L P 1961 Zh. Eksp. Teor. Fiz. 40, 646 [English Transl. 1961 Sov. Phys. JETP 13 451]

[12] Olshanii M 1998 Phys. Rev. Lett. 81938

[13] Chiofalo M L and Tosi M P 2000 Phys. Lett. A 268406

[14] Landau L D and Lifsits E M 1991 Mechanics, Course of Theoretical Physics, vol. 3 (Pergamon Press: Oxford); Arnold V I 1990 Mathematical Methods of Classical Mechanics (Springer: Berlin). 
[15] Andrews M R, Townsend C G, Miesner H J, Drufee D S, Kurn D M, and Ketterle W 1997 Science 275637

[16] Fribel S, D'Andrea C, Walz J, Weitz M, and Hänsch T W 1998 Phys. Rev. A 57 R20

[17] Jauregui R, Poli N, Roati G, and Modugno G 2001 Phys. Rev. A 64033403

[18] Kevrekidis P G, Bishop A R, and Rasmussen K O 2000 J. Low Temp. Phys. 120205

[19] Salasnich L 2000 Phys. Lett. A 266 187; Salasnich L 2000 Progr. Theor. Phys. Suppl. 139414

[20] Cohen-Tannouji C 1998 Rev. Mod. Phys. 70707 


\section{FIGURES}

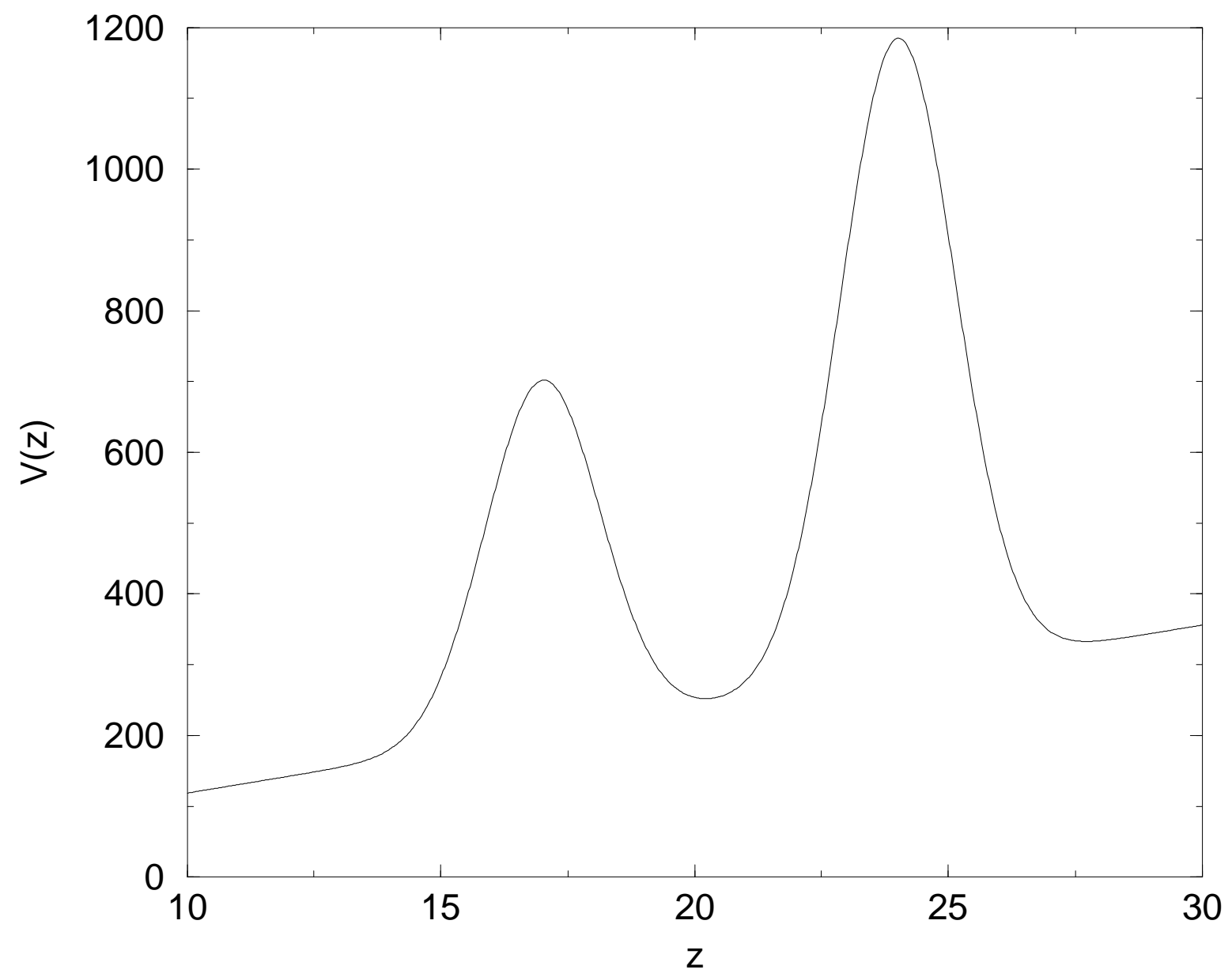

FIG. 1. Vertical axial external potential $V(z)$ of Eq. (2). Scaled parameters of the potential: $V_{1}=300, V_{2}=900, z_{1}=17, z_{2}=24, \sigma=1.6$. Length $z$ in units $a_{z}=\left(\hbar / m \omega_{z}\right)$, where $\omega_{z}=\omega_{\perp} / 10$ with $\omega_{\perp}=2 \pi \mathrm{kHz}$. Energy in units $\hbar \omega_{z}$. 

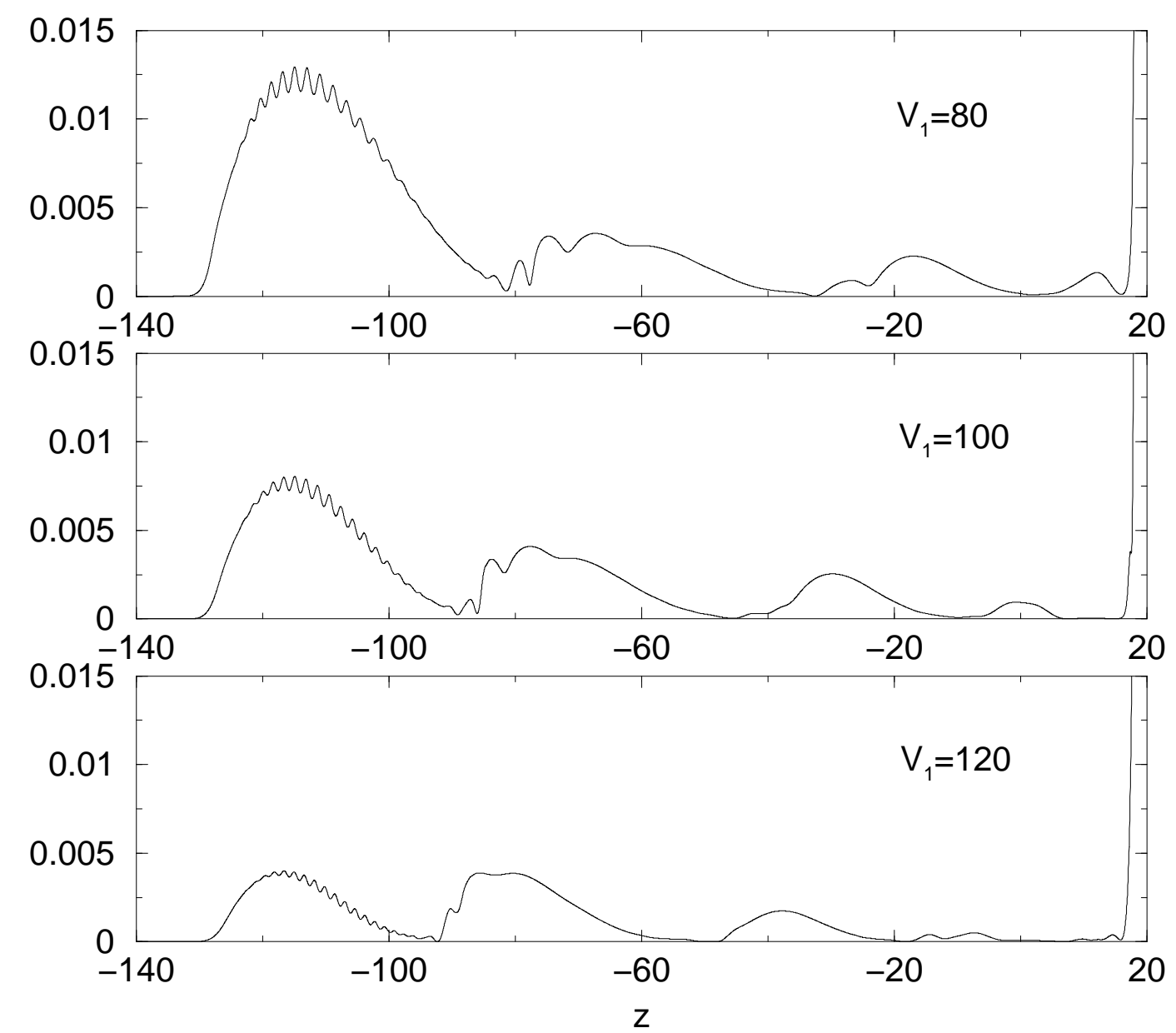

FIG. 2. Axial density profile of Bose condensed ${ }^{23} \mathrm{Na}$ atoms tunneling through the potential well (Eq. (5)), as obtained by solving NPSE. Time: $t=4.8$. Number of ${ }^{23} \mathrm{Na}$ atoms: $N=10^{4}$. Scattering length: $a_{s}=30 \AA$. Potential well parameters: $z_{1}=17, z_{2}=24$ and $V_{2}=900$. Three values of $V_{1}$ correspond to three values of the effective chemical potential $\mu_{e f f}$ and of the period $\tau_{\text {osc }}$ of small oscillations around the minimum of the potential well: $\mu_{\text {eff }}=53.91$ and $\tau_{\text {osc }}=1.21$ (top), $\mu_{e f f}=54.23$ and $\tau_{o s c}=1.17$ (middle), $\mu_{e f f}=54.55$ and $\tau_{o s c}=0.90$ (bottom). Length $z$ in units $a_{z}=\left(\hbar / m \omega_{z}\right)$, where $\omega_{z}=\omega_{\perp} / 10$ with $\omega_{\perp}=2 \pi \mathrm{kHz}$; energy in units $\hbar \omega_{z}$ and time in units $\omega_{z}^{-1}$ 

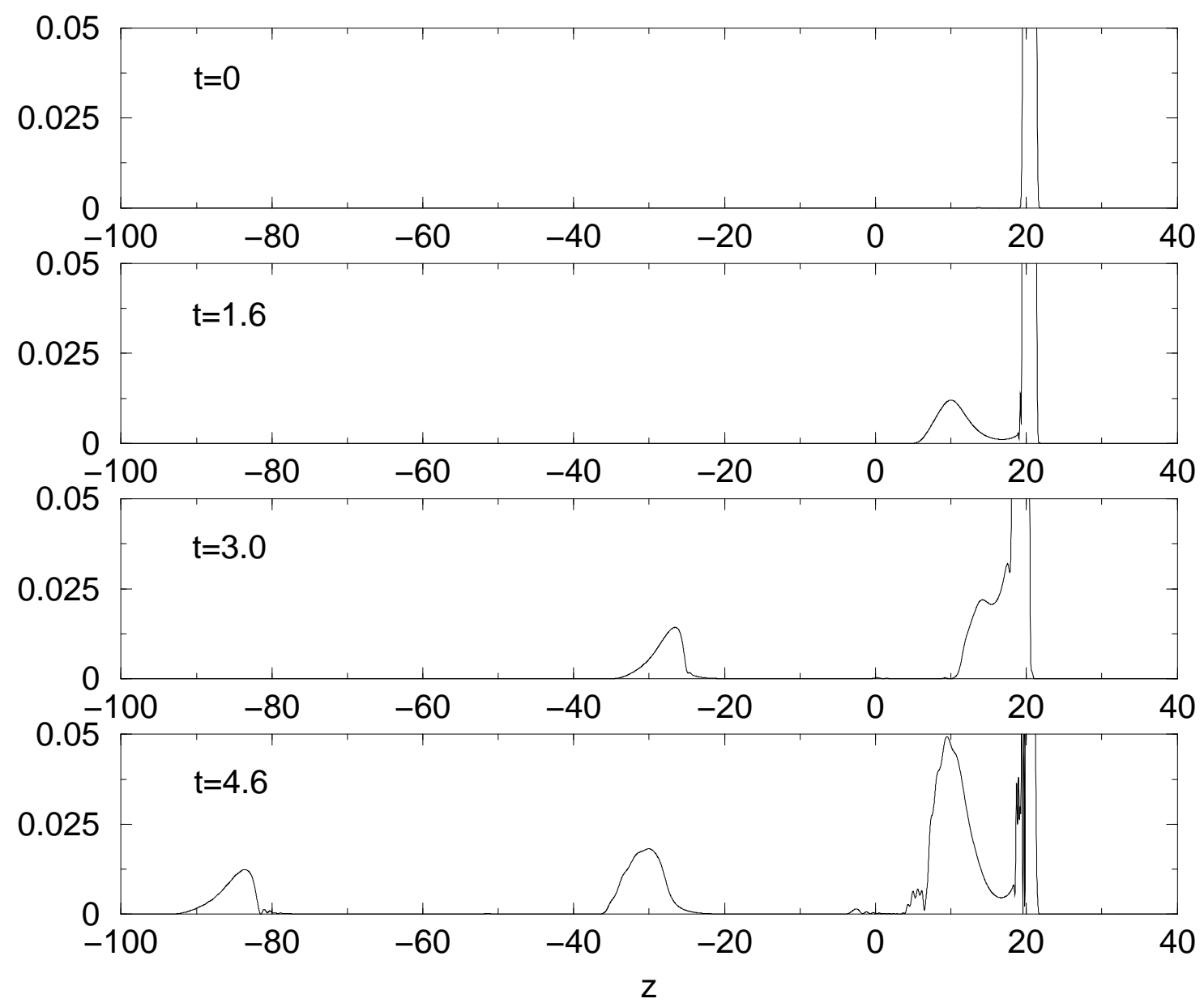

FIG. 3. Axial density profile of Bose condensed ${ }^{23} \mathrm{Na}$ atoms tunneling through the potential well (Eq. (2)) with oscillating barrier: $V_{1}(t)=V_{a}+V_{b} \sin \left(\frac{2 \pi}{\tau} t\right), V_{a}=300$ and $V_{b}=200$. Results obtained by solving NPSE. Period of oscillation: $\tau=1.5$. Effective chemical potential of the initial condensate: $\mu_{e f f}=57.43$. Number of ${ }^{23} \mathrm{Na}$ atoms: $N=10^{4}$. Scattering length: $a_{s}=30 \AA$ A . Note that $P_{T}=0.26$ with $V_{1}(t)=V_{a}-V_{b}=100$. Units as in Fig. 2. 


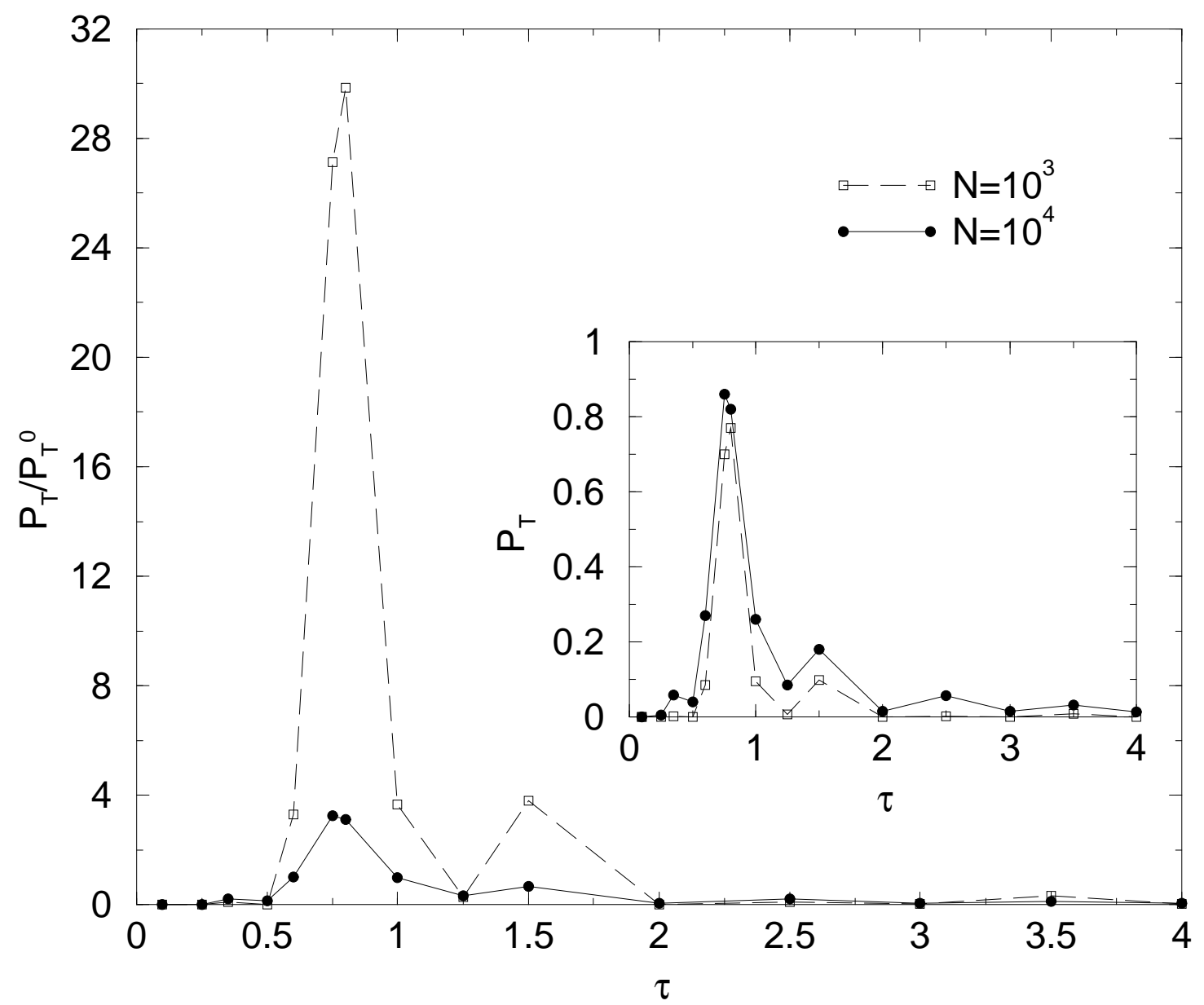

FIG. 4. Tunneling ratio $P_{T} / P_{T}^{0}$ at $t=5$ as a function of the period $\tau$ of oscillation of the lower-lying Gaussian barrier (Eq. (3)), as obtained by solving NPSE. $V_{1}(t)=V_{a}+V_{b} \sin \left(\frac{2 \pi}{\tau} t\right)$, $V_{a}=300$ and $V_{b}=200$ (Eq. (6)). The period $\tau_{\text {osc }}$ of small oscillations around the minimum of the potential well with $V_{a}=300$ and $V_{b}=0$ is $\tau_{o s c}=0.84 . P_{T}^{0}$ is the tunneling probability with $V_{1}=V_{a}-V_{b}=100$. Effective chemical potential of the initial condensate: $\mu_{e f f}=27.15$ for $N=10^{3}, \mu_{e f f}=57.43$ for $N=10^{4} . N$ is the number of ${ }^{23} \mathrm{Na}$ atoms. Scattering length: $a_{s}=30$ A. Inset: tunneling probability $P_{T}$ at $\mathrm{t}=5$ as a function of $\tau$. Units as in Fig. 2 . 

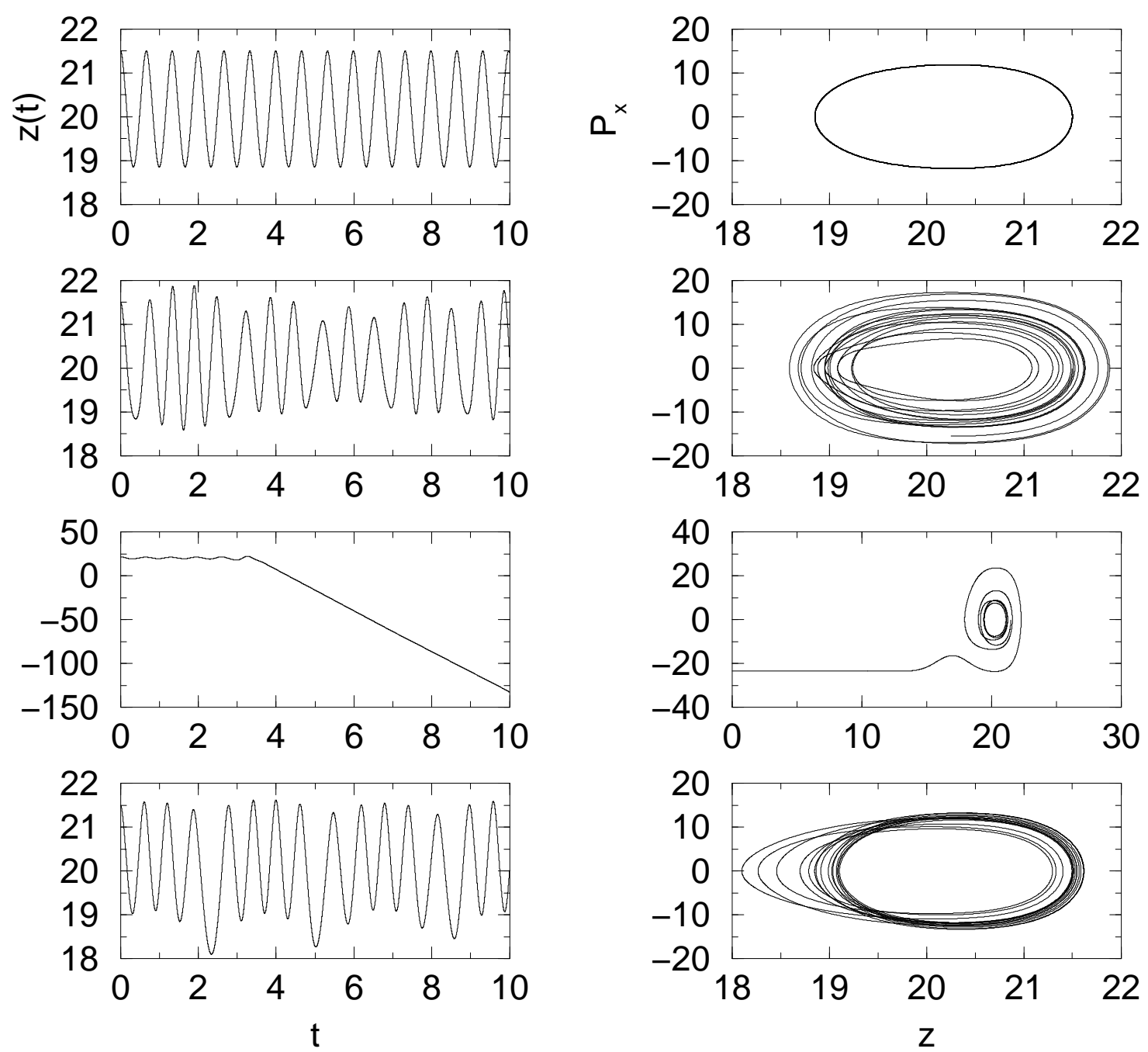

FIG. 5. Axial coordinate $z(t)$ (left) and phase-space portrait $\left(z, p_{z}\right)$ with $p_{z}=\dot{z}$ (right) of a classical particle under the action of the potential $V(z)$ given by Eq. (2) with its parameters as in Fig. 4. From top to bottom: (a) $V_{b}=0$ and $\tau=$ anything; (b) $V_{b}=200, \tau=0.5$; (c) $V_{b}=200$ and $\tau=0.75 ;(\mathrm{d}) V_{b}=200$ and $\tau=3$. Units as in Fig. 2. 


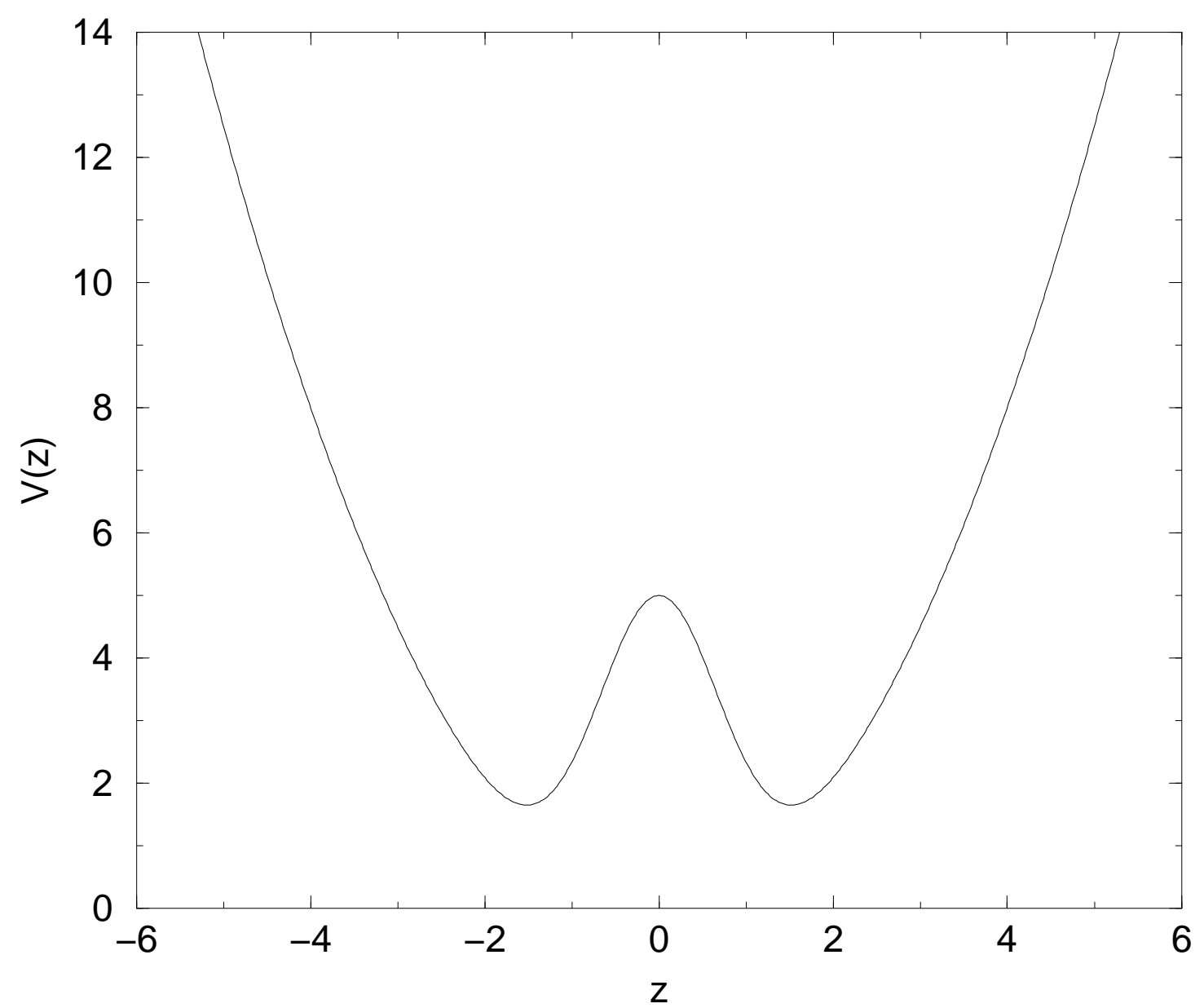

FIG. 6. Horizontal axial double-well potential $V(z)$ given by Eq. (4). Scaled parameters of the potential: $V_{1}=5, V_{2}=0, \sigma=1$. Length $z$ in units $a_{z}=\left(\hbar / m \omega_{z}\right)$ and energy in units $\hbar \omega_{z}$. 

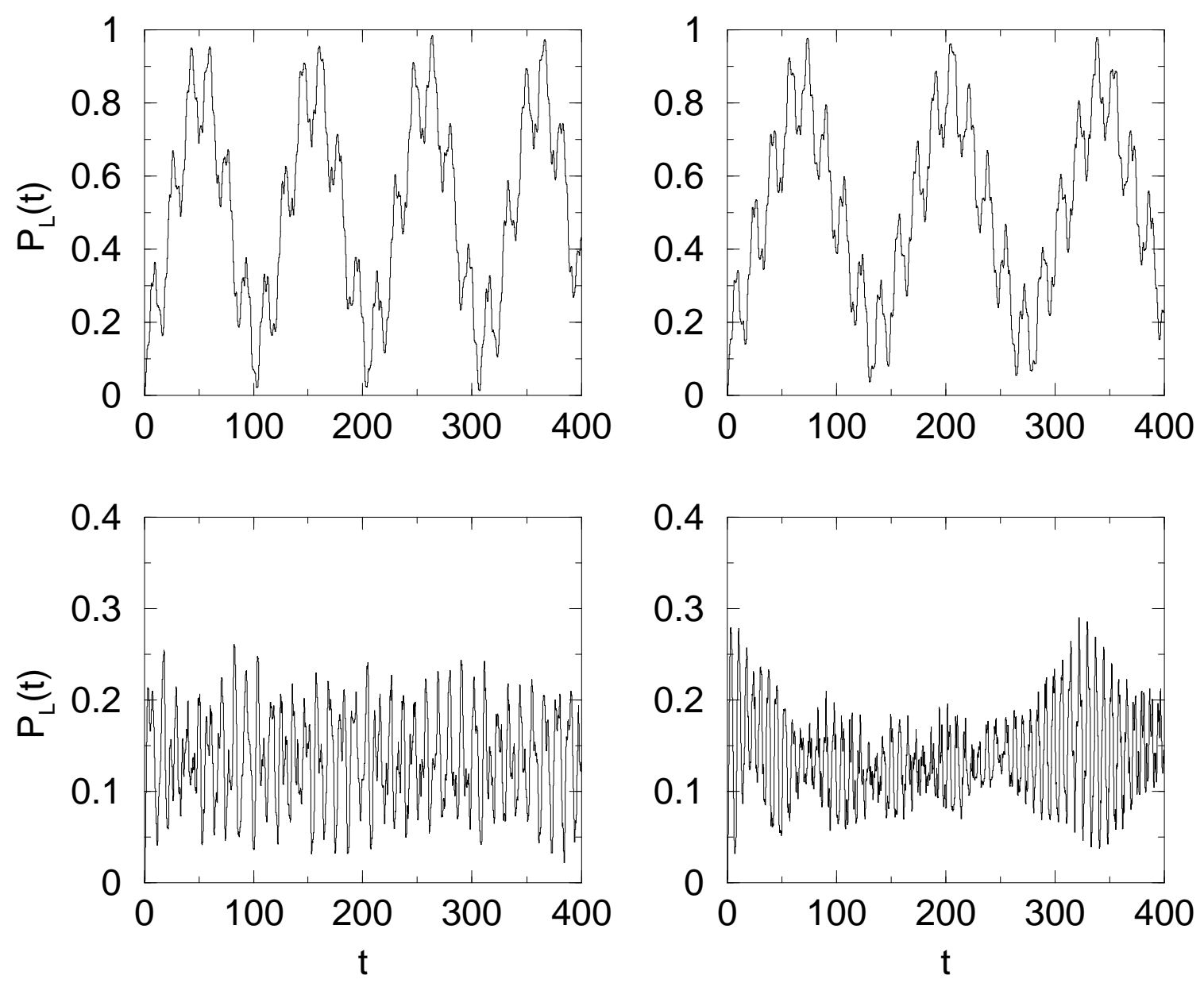

FIG. 7. Fraction $P_{L}(t)$ of Bose condensed ${ }^{23} \mathrm{Na}$ atoms in the left well obtained by solving NPSE where the external potential is given by Eq. (3) with $V_{1}=6.5$ and $V_{2}=0$. From top to bottom and from left to right: (a) $N a_{s} / a_{z}=0$; (b) $N a_{s} / a_{z}=0.014$; (c) $N a_{s} / a_{z}=0.07$; (d) $N a_{s} / a_{z}=0.14$. Length $z$ in units $a_{z}=\left(\hbar / m \omega_{z}\right)$, where $\omega_{z}=\omega_{\perp} / 10$ with $\omega_{\perp}=2 \pi \mathrm{kHz}$. Energy in units $\hbar \omega_{z}$ and time in units $\omega_{z}^{-1}$. 


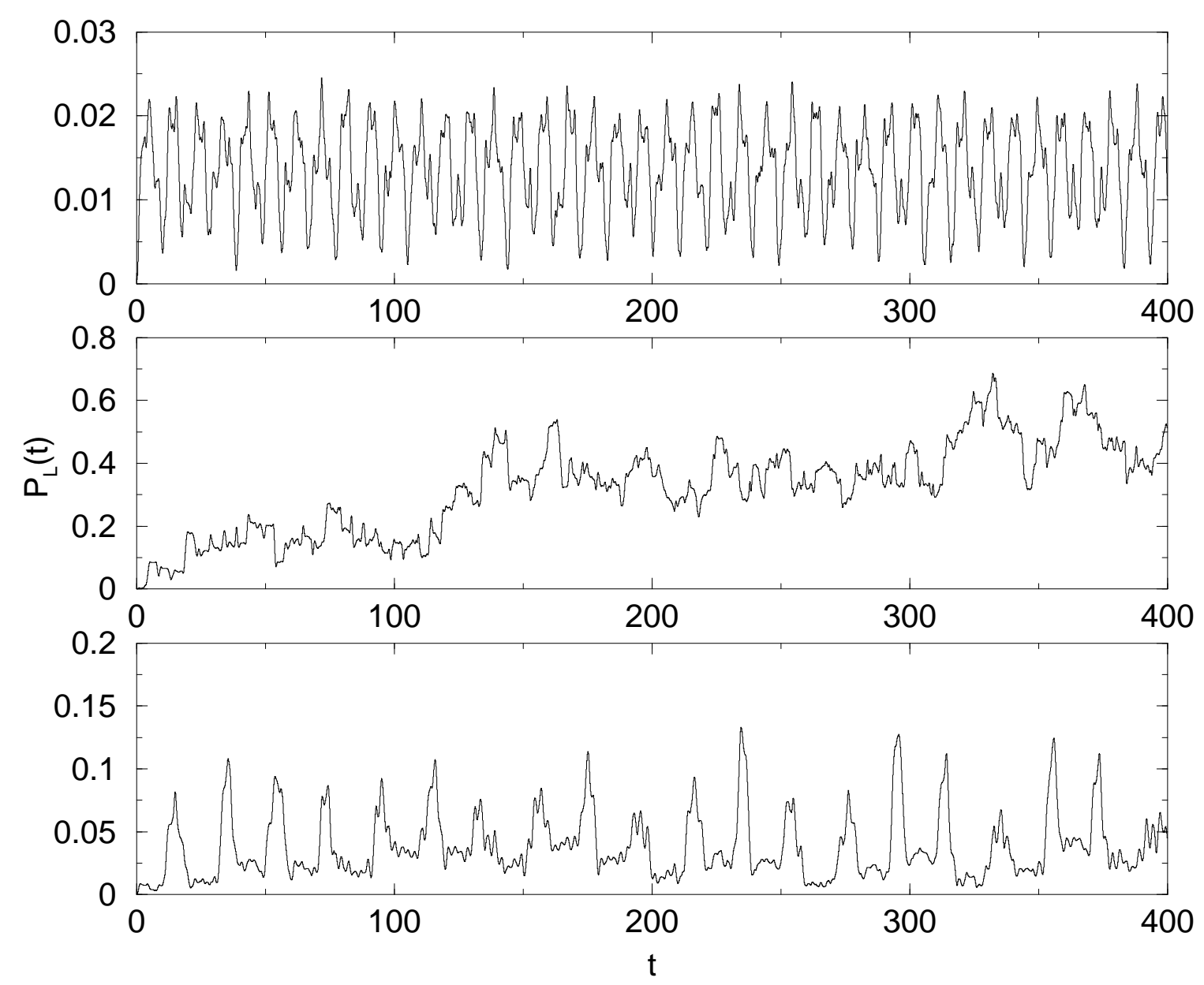

FIG. 8. Fraction $P_{L}(t)$ of Bose condensed ${ }^{23} \mathrm{Na}$ atoms in the left well obtained by solving NPSE where the external potential is given by Eq. (3) with $V_{1}=6.5$. From top to bottom: (a) $V_{2}=0$ and $\tau=$ anything; (b) $V_{2}=2$ and $\tau=\tau_{S T} / 2$; (c) $V_{2}=2$ and $\tau=2 \tau_{S T}$; where $\tau_{S T}$ is the period of unperturbed MQST oscillation. Units as in Fig. 7. 

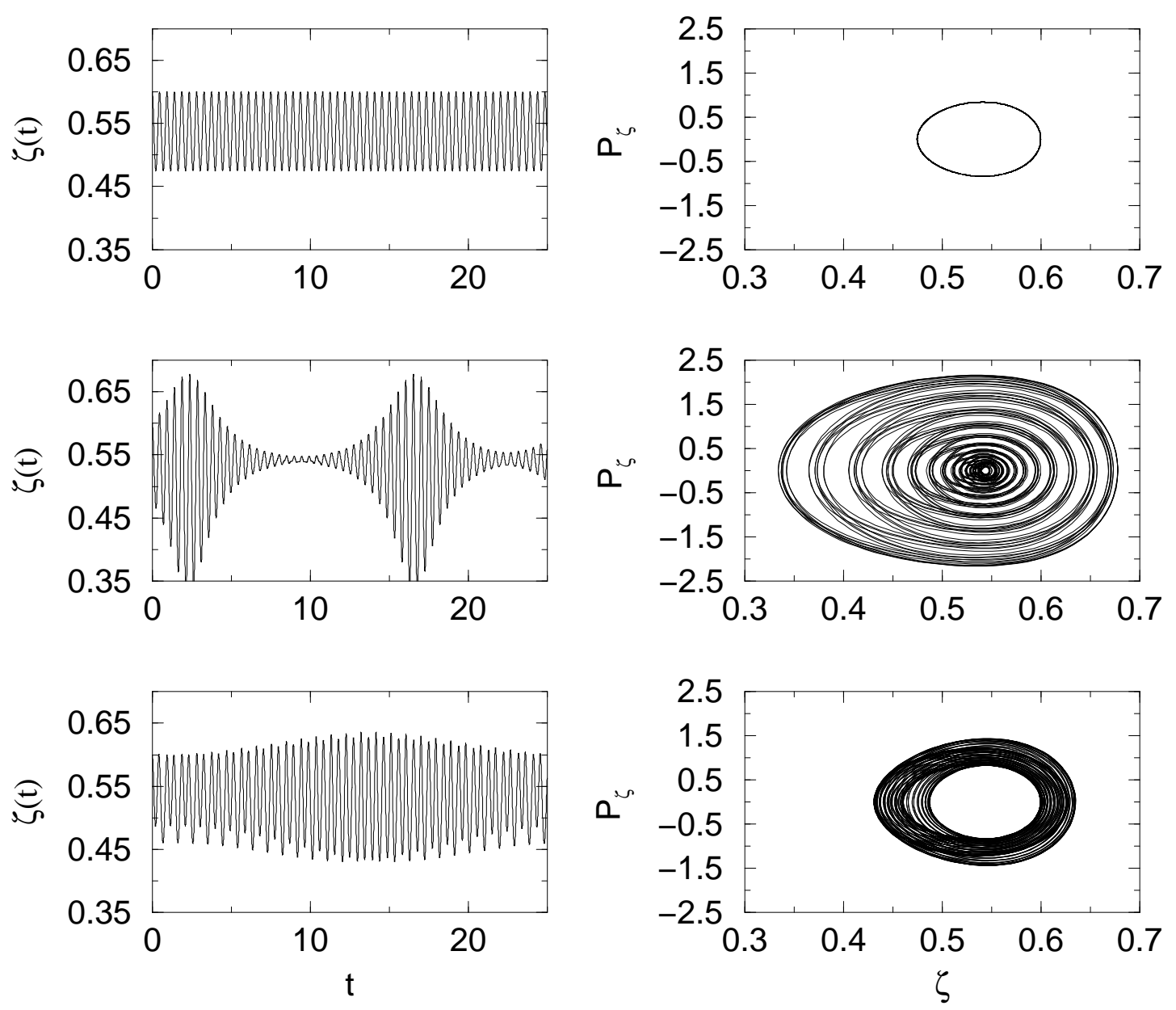

FIG. 9. Fractional population imbalance $\zeta(t)$ of the condensate in the two wells (left) and its phase-space portrait $\left(\zeta, p_{\zeta}\right)$ with $p_{\zeta}=\dot{\zeta}$ (right), as obtained by solving Eq. (6). Initial conditions: $\zeta(0)=0.6, \phi(0)=0 . \Lambda=\Lambda_{0}(1+\epsilon \sin (2 \pi t / \tau))$ where $\Lambda_{0}=25$. From top to bottom: (a) $\epsilon=0$ and $\tau=$ anything; (b) $\epsilon=0.1$ and $\tau=\tau_{S T} / 2$; (c) $\epsilon=0.1$ and $\tau=2 \tau_{S T}$; where $\tau_{S T}$ is the period of unperturbed MQST oscillations. 

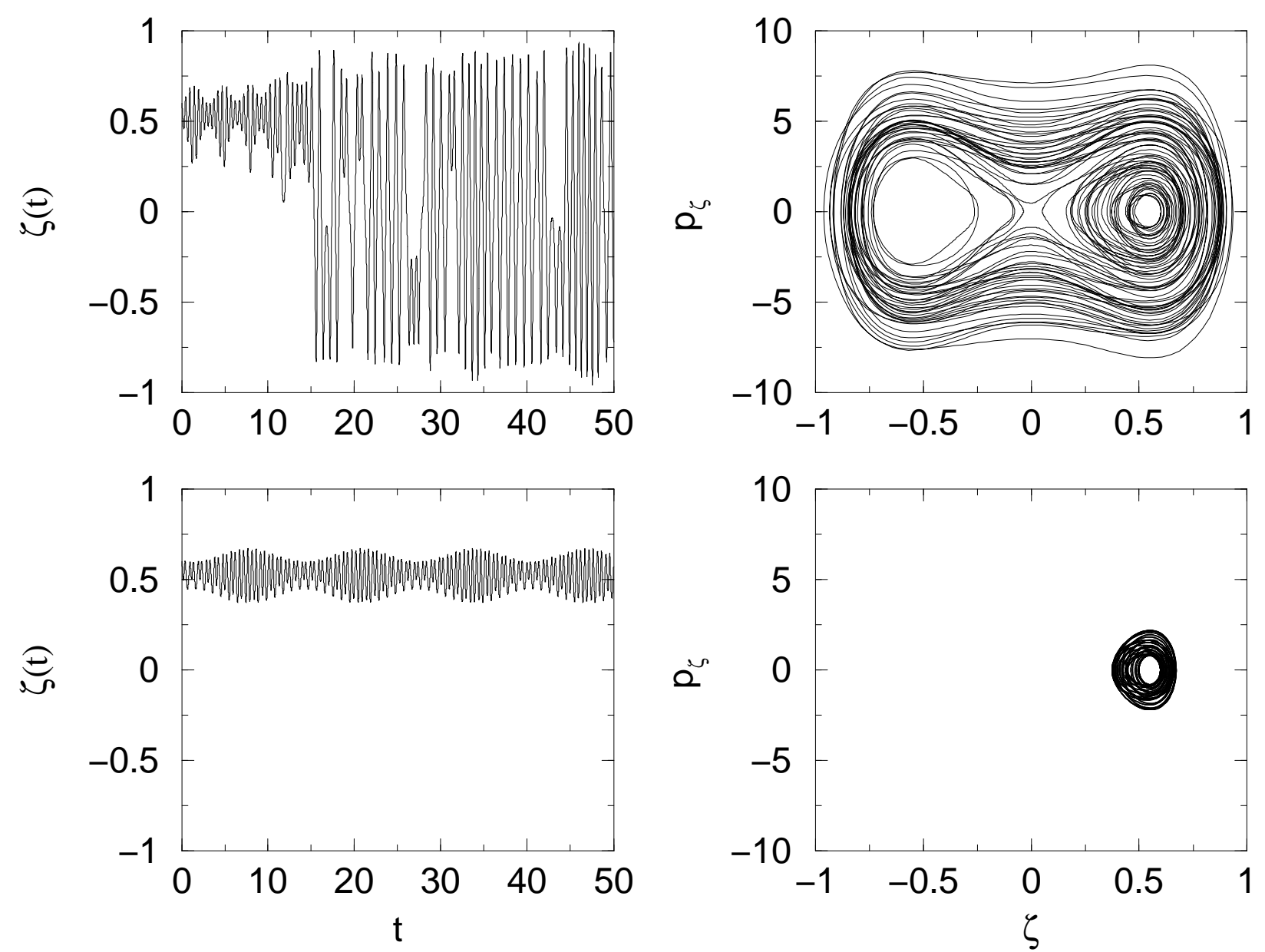

FIG. 10. Fractional population imbalance $\zeta(t)$ of the condensate in the two wells (left) and its phase-space portrait $\left(\zeta, p_{\zeta}\right)$ with $p_{\zeta}=\dot{\zeta}$ (right), as obtained by solving Eq. (6). Initial conditions: $\zeta(0)=0.6, \phi(0)=0 . \Lambda=\Lambda_{0}(1+\epsilon \sin (2 \pi t / \tau))$ where $\Lambda_{0}=25$ and $\epsilon=0.2$. From top to bottom: (a) $\tau=\tau_{S T} / 2$; (b) $\tau=2 \tau_{S T}$; where $\tau_{S T}$ the period of unperturbed MQST oscillations. 